\title{
Regulating the CCNB1 gene can affect cell proliferation and apoptosis in pituitary adenomas and activate epithelial-to-mesenchymal transition
}

\author{
BIN LI $^{1 *}$, HAI-BO ZHU ${ }^{1 *}$, GUI-DONG SONG ${ }^{2}$, JIAN-HUA CHENG ${ }^{1}$, \\ CHU-ZHONG LI ${ }^{2}$, YA-ZHUO ZHANG ${ }^{2}$ and PENG ZHAO ${ }^{1}$ \\ ${ }^{1}$ Neurosurgical Department, Beijing Tiantan Hospital, Capital Medical University; \\ ${ }^{2}$ Department of Cell and Biology, Beijing Neurosurgical Institute, Beijing 100070, P.R. China
}

Received March 7, 2019; Accepted August 6, 2019

DOI: 10.3892/ol.2019.10847

\begin{abstract}
The aim of the present study was to investigate the role and potential regulatory mechanisms of cyclin $\mathrm{B} 1$ (CCNB1) in the proliferation, apoptosis and epithelial-to-mesenchymal transition (EMT) in pituitary adenomas. A total of 24 specimens were included in the present study. The expression levels of CCNB1 protein in two normal pituitary and 22 pituitary adenoma tissues were determined by western blotting. CCNB1 was knocked-down by lentiviral-mediated infection of short hairpin RNA (shRNA) in GH3 and MMQ cell lines. The proliferation, cell cycle and apoptosis of GH3 and MMQ cell lines were detected using a Cell Counting Kit-8 and flow cytometer. Reverse transcription-quantitative PCR was utilized to detect the expression level of CCNB1 gene and EMT markers. In the present study, resveratrol (RES) was used as an inhibitor of CCNB1. The protein expression level of CCNB1 in pituitary adenomas was higher than that in normal pituitary tissue, as assessed by western blot analysis. In addition, the expression level of CCNB1 in invasive pituitary adenomas was higher when comparing invasive pituitary adenomas and non-invasive pituitary adenomas. Knockdown of CCNB1 resulted in significant decreases in cell viability and proliferation, arrested cell cycle at the $\mathrm{G}_{2} / \mathrm{M}$ phase and increased apoptosis. In addition, knockdown of CCNB1 significantly decreased the expression levels of the mesothelial cell marker $\mathrm{N}$-cadherin $(\mathrm{P}<0.001)$, but significantly increased the expression levels of the epithelial cell markers E-cadherin $(\mathrm{P}<0.01)$ and p120-catenin $(\mathrm{P}<0.001)$. Further analyses identified that RES inhibited the expression
\end{abstract}

Correspondence to: Professor Peng Zhao, Neurosurgical Department, Beijing Tiantan Hospital, Capital Medical University, 119 South Fourth Ring West Road, Fengtai, Beijing 100070, P.R. China

E-mail: zhaopeng@ccmu.edu.cn

*Contributed equally

Key words: cyclin B1, pituitary adenomas, proliferation, apoptosis, epithelial-to-mesenchymal transition, resveratrol level of CCNB1, and RES treatment exhibited a similar effect as CCNB1 shRNA infection. The present study suggested that suppressing the expression level of CCNB1 could regulate the proliferation and apoptosis of pituitary tumor cells and alter the expression level of various EMT markers. In addition, RES treatment could be used as an inhibitor of CCNB1. The present study also identified the molecular mechanisms underlying CCNB1 role in EMT.

\section{Introduction}

Pituitary adenoma is one of the most common neuroendocrine tumors (1). The incidence rate is $3.47 / 100,000$ in the United States in 2015, which corresponds to $10-25 \%$ of tumors of the central nervous system $(1,2)$. Although most pituitary adenomas are histologically benign, 34-60\% of the lesions that remain destroy the dura, periosteum and even the bone, exhibiting malignant and invasive characteristics (3). The invasion and migration features of pituitary adenomas result in difficulties in surgical treatment and postoperative recurrence (4). The diagnosis of invasive pituitary adenoma (IPA) is based on comprehensive imaging, intraoperative investigation and postoperative pathology. At present, there is a lack of specific molecular biological indicators, which is a difficult issue to overcome in clinical treatment (5).

A previous study demonstrated that CCNB1 protein, encoded by the CCNB1 gene, belongs to the cyclin superfamily and is expressed in almost all tissues in humans (6). CCNB1 can form a complex with p34, also known as cdc2, forming the maturation-promoting factor, and is necessary for proper control of the $G_{2} / M$ transition phase of the cell cycle (7). In normal cells, CCNB1 is primarily expressed in the late $S$ stage, increases significantly in the $G_{2}$ phase, and reaches its peak value at $\mathrm{M}$ stage $(8,9)$. CCNB1 is highly expressed in numerous different types of human tumors, including breast cancer, cervical cancer, lung cancer, esophageal squamous cell carcinoma and melanoma (10-15), indicating its potential role in cancer transformation and progression. In line with previous findings, CCNB1 identified in the integrated analysis was closely related to the tumorigenesis of pituitary adenomas (16). Further research found that upregulation of 
cyclin B1 has potential roles in the invasiveness of pituitary adenomas (17). However, the specific molecular mechanism underlying CCNB1 function remains unclear.

EMT is supposed to be responsible for increased invasion and metastasis in epithelial cancer cells (18). The activation of EMT facilitates cancer invasion and metastasis, and accelerates cancer progression $(19,20)$. It has been reported that CCNB1 may be involved in the processes of EMT and metastasis (21). Therefore, there may be a certain association between the cyclin family and EMT activation in pituitary tumor cell transformation and tumor progression.

Resveratrol (RES) is a common drug used to inhibit the proliferation of a number of cancers. In addition, RES can affect cell cycle and apoptosis by interacting with various key genes (22-24). RES may inhibit the expression of CCNB1 and CDK1 in tumor cells, and can induce cells to stop at the $\mathrm{G}_{2} / \mathrm{M}$ stage (25). Therefore, due to its potential in suppressing cell proliferation, RES can be used as a cell cycle inhibitor.

The aim of the present study was to further investigate the role and potential regulatory mechanism of CCNB1 in the proliferation and apoptosis of pituitary adenomas. In the present study, the expression level of CCNB1 was found to influence the EMT process and cell function was found to be affected following lentiviral-mediated CCNB1 knockdown in GH3 and MMQ cell lines. RES was found to act as an inhibitor of proliferation, and RES treatment was able to affect the expression levels of CCNB1 in pituitary tumor cells.

\section{Materials and methods}

Patients and specimens. A total of 24 samples were included in the present study. Twenty-two pituitary adenoma specimens were obtained during neurological surgery between September 2016 and December 2017 from patients at the Beijing Tiantan Hospital, Capital Medical University (Beijing, China). In addition, two normal pituitary glands that were donated by two subjects (deceased for other causes) were used as control. A total of 10 women and 12 men with pituitary adenoma were included in the present study. The mean age of the patients was 40.2 years (range, 15-74 years) at the time of surgery. One of the normal pituitary glands was from a 49-year-old female and the other was from a 35-year-old male. Patients were divided into invasive pituitary adenoma (IPA) and non-invasive pituitary adenoma (NIPA) groups according to Hardy-Wilson $(26,27)$ and Knosp classification (28). According to the Knosp classification, $0-1$ grade pituitary adenoma belongs to the NIPA groups and 2-4 grade pituitary adenoma belongs to IPA groups. In the Hardy-Wilson classification, pituitary adenoma of grade I-II and stage A-B belongs to the NIPA groups, grade III-IV and stage C-E belongs to the IPA groups. Based on the preoperative endocrinological tests and postoperative pathological results, which were derived from routine pathological examination of pituitary adenomas by pathologist Professor Gui-Lin Li (Department of Pathology, Beijing Tiantan Hospital), all the specimens were considered as non-functional adenomas.

First, the expression level of CCNB1 was investigated in 14 pituitary adenoma tissue specimens and a normal pituitary gland. This group was studied to detect the expression level of CCNB1 in normal pituitary and pituitary adenoma.
In addition, four NIPA and four IPA tissue specimens with a normal pituitary were examined, which were not included in the 14 aforementioned samples. This group was examined to detect the expression of CCNB1 in IPA and NIPA samples. The pituitary adenoma samples were quickly frozen in liquid nitrogen within $30 \mathrm{~min}$ of surgery. All patients enrolled were followed up for 1-2 years. The present study was approved by the Beijing Tiantan Hospital Ethics Committee, and written informed consent was obtained from every participant.

Cell culture. Rat pituitary tumor cell lines GH3 and MMQ (Institute of Basic Medical Sciences, Chinese Academy of Sciences) were maintained in F-12 (Gibco; Thermo Fisher Scientific, Inc.), supplemented with $15 \%$ horse serum (HS; Beijing Solarbio Science and Technology Co., Ltd.), 2.5\% FBS (Gibco; Thermo Fisher Scientific, Inc.), $100 \mathrm{U} / \mathrm{ml}$ penicillin and $100 \mu \mathrm{g} / \mathrm{ml}$ streptomycin under $5 \% \mathrm{CO}_{2}$, at $37^{\circ} \mathrm{C}$ in a humidified incubator. Cells were regularly passaged to maintain exponential growth.

Lentiviral CCNB1 short hairpin RNA (shRNA) recombinant vector production and transfection. Based on the gene sequence of CCNB1 in GenBank (Gene ID, 25203; https://www.ncbi. nlm.nih.gov/gene/25203), primers of CCNB1 shRNA $(0.5 \mu \mathrm{M})$ and negative control $(0.5 \mu \mathrm{M})$ were cloned into a pGMLV-SC5 vector (Genomeditech Co., Ltd.), which carried the green fluorescent protein (GFP) gene. The sequences used to construct the short hairpin targeting CCNB1 (CCNB1-shRNA) and the respective negative control are presented in Table I.

The 293T cells (American Type Culture Collection) were selected for packaging and titer measurement of the lentivirus. GH3 and MMQ cells were transferred to each well of a 6 -well culture plate $24 \mathrm{~h}$ before transfection. When cells reached $70-80 \%$ confluence, the constructed lentiviral vector and its auxiliary packaging original vector plasmid for control were co-transfected into 293T cells using HG transgene reagent (Genomeditech Co., Ltd.). The following groups were analyzed: i) non-infected (Con); ii) negative control shRNA-infected (NC); iii) CCNB1 shRNA-infected (shRNA). After cell culture for 5 days, the number of fluorescent cells in the wells was observed under a fluorescence microscope (x100 magnification) and the infection rate (the number of fluorescent cells/by the total number of cells) was calculated using Image Pro Plus software (v6.0; Media Cybernetics, Inc). CCNB1-shRNA lentivirus titer was set in $5 \times 10^{8} \mathrm{TU} / \mathrm{ml}$, and normal control-shRNA lentivirus titer was in $6 \times 10^{8} \mathrm{TU} / \mathrm{ml}$.

Reverse transcription-quantitative PCR (RT-qPCR) assay. Total RNA was extracted from the cell lines and pituitary adenoma tissue samples using TRIzol ${ }^{\circledR}$ reagent (Invitrogen; Thermo Fisher Scientific, Inc.), and RNA was reverse transcribed to cDNA using the High-Capacity cDNA Reverse Transcription Kit (Thermo Fisher Scientific, Inc.) according to the manufacturer's protocol. The reverse transcription conditions were: $25^{\circ} \mathrm{C}$ for $10 \mathrm{~min}, 37^{\circ} \mathrm{C}$ for $120 \mathrm{~min}$ and $85^{\circ} \mathrm{C}$ for $5 \mathrm{~min}$. Primers were designed using Primer software (version 5.0; Premier Biosoft International) based on gene sequences from the Genbank database for CCNB1 (Table II). The qPCR was performed using PowerUp ${ }^{\mathrm{TM}} \mathrm{SYBR}^{\mathrm{TM}}$ green 
Table I. Primers for CCNB1-shRNA.

\begin{tabular}{ll}
\hline Oligonucleotide & \multicolumn{1}{c}{ Sequence (5'-3') } \\
\hline CCNB1-shRNA & F: gatccGCCTGAGCCTGAACCTGTTATTTCAAGAGAATAACAGGTTCAGGCTCAGGCTTTTTTg \\
& R: aattcAAAAAAGCCTGAGCCTGAACCTGTTATTCTCTTGAAATAACAGGTTCAGGCTCAGGCg \\
Negative control & F: gatcTGTTCTCCGAACGTGTCACGTTTCAAGAGAACGTGACACGTTCGGAGAATTTTTC \\
& R: aattgAAAAAATTCTCCGAACGTGTCACGTTCTCTTGAAACGTGACACGTTCGGAGAACa \\
\hline
\end{tabular}

CCNB1, cyclin B1; shRNA, short hairpin RNA; F, forward; R, reverse.

master mix on a Pharmaceutical Analytics QuantStudio ${ }^{\mathrm{TM}} 5$ Real-Time PCR System (both Thermo Fisher Scientific, Inc.). PCR conditions were as follows: Initial denaturation at $95^{\circ} \mathrm{C}$ for $2 \mathrm{~min}$, followed by 40 cycles of $95^{\circ} \mathrm{C}$ for $1 \mathrm{sec}$ and $60^{\circ} \mathrm{C}$ for $30 \mathrm{sec}$. The PCR results were verified by the melting curve. The data were normalized to the housekeeping gene GAPDH and counted using the $2^{-\Delta \Delta C q}$ method (29).

Protein extraction and western blot analysis. The tissues and treated GH3 and MMQ cells were lysed with RIPA buffer containing a 50X protease inhibitor (both Applygen Technologies Inc.) cocktail. The concentration of protein was determined using a BCA Protein Assay kit (Beijing Solarbio Science \& Technology, Co., Ltd.). Proteins (30 $\mu \mathrm{g}$ per lane) were separated via SDS-PAGE (10\% gel) and transferred to PVDF membranes (EMD Millipore). The membranes were incubated with the primary antibodies anti-CCNB1 $(1: 1,000$; cat. no. 12231$)$, anti- $\beta$-actin $(1: 1,000$; cat. no. 4970$)$ and anti-GAPDH (1:1,000; cat. no. 5174) (all from Cell Signaling Technology, Inc.). After washing with TBST three times, the membrane was incubated with anti-rabbit IgG HRP-linked antibody (1:4,000; cat. no. 7074; Cell Signaling Technology, Inc.) for $1 \mathrm{~h}$ at room temperature and then washed with TBST three times. The ECL chemiluminescence reagent (Santa Cruz Biotechnology, Inc.) was used for detection. $\beta$-actin and GAPDH were used as internal controls and the grey values of the protein bands were quantified with the Image J software (version 1.8; National Institutes of Health).

Cell Counting Kit-8 (CCK-8) assay. Cell proliferation was analyzed with a CCK-8 kit (Dojindo Molecular Technologies, Inc.) according to the manufacturer's protocol. Following treatment, GH3 and MMQ cells were seeded and grown in 96-well plates. A solution containing $90 \mu \mathrm{l}$ fresh F-12 medium [(Gibco; Thermo Fisher Scientific, Inc.) supplemented with $15 \%$ HS (Beijing Solarbio Science and Technology Co., Ltd.), 2.5\% FBS (Gibco; Thermo Fisher Scientific, Inc.)] and $10 \mu \mathrm{l} \mathrm{CCK-8}$ solution were added to each sample at 24, 48, 72 and $96 \mathrm{~h}$. Subsequently, cells were incubated at $37^{\circ} \mathrm{C}$ for $1-4 \mathrm{~h}$. The optical density value was measured using a microplate reader (Varioskan Flash; Thermo Fisher Scientific, Inc.) at the wavelength of $450 \mathrm{~nm}$.

Flow cytometric analysis of cell cycle distribution. To analyze cell cycle distribution, the treated cells were digested by pancreatin and washed with ice-cold PBS, then suspended in $1 \mathrm{ml}$ ice-cold $70 \%$ ethanol. The cells were washed with ice-cold PBS prior to staining and resuspended in $1 \mathrm{ml}$ of
Vindelov's propidium iodide (PI) at room temperature for 30 min in the dark. The DNA content of cells was analyzed using a flow cytometer (BD Accuri ${ }^{\mathrm{TM}}$ C6; BD Biosciences). Cell cycle distribution was analyzed as a typical DNA content histogram using BD CellQuest ${ }^{\mathrm{TM}}$ cell cycle analysis software (version 5.1; BD Biosciences).

Annexin V-FITC/PI staining. Cellular apoptosis was evaluated via Annexin V-FITC apoptosis detection kit (Sigma-Aldrich; Merck $\mathrm{KGaA})$. The treated cells were collected by centrifugation $\left(37^{\circ} \mathrm{C}\right.$ for $5 \mathrm{~min}$ at $\left.500 \mathrm{x} \mathrm{g}\right)$ and suspended in $500 \mu \mathrm{l}$ 10X binding buffer (Sigma-Aldrich; Merck KGaA), and $5 \mu 1$ Annexin V-FITC and $5 \mu \mathrm{l}$ PI were added. The cells were incubated at room temperature for $30 \mathrm{~min}$ in the dark. The cells stained with Annexin V-FITC and PI were detected using a flow cytometer (BD FACS Calibur; BD Biosciences). Flow cytometry of Annexin V-FITC/PI apoptosis assay was determined to quantify necrotic, early apoptotic, late apoptotic and viable cells. The cytometric data were analysed using WinMDI version 2.9 (The Scripps Research Institute).

EMT-associated marker assay. In order to evaluate the activation of the EMT by CCNB1, the associated markers were analyzed via RT-qPCR. The epithelial cell markers tested were E-cadherin and p120-catenin, and the mesothelial cell marker was $\mathrm{N}$-cadherin. The treated GH3 and MMQ cells were seeded at a density of $2 \times 10^{4}$ cells/well in 6-well plates and treated with or without shRNA or inhibitor for 24,48 and $72 \mathrm{~h}$. The primers used are listed in Table II. The RT-qPCR was performed following the aforementioned protocol.

RES inhibition assay. For the in vitro CCNB1 inhibitor experiments, RES (Beijing Solarbio Science \& Technology, Co., Ltd.) was dissolved in dimethyl sulfoxide (DMSO; Beijing Solarbio Science \& Technology, Co., Ltd.) and added to the F-12 culture medium, based on the research by Joe et al (30). Briefly, cells were treated with $0.2 \%$ DMSO (negative control) and RES inhibitor (100 and $300 \mu \mathrm{M})$ at $37^{\circ} \mathrm{C}$ in a humidified incubator with $5 \% \mathrm{CO}_{2}$. After $48 \mathrm{~h}$ of treatment, both adherent and floating cells were harvested for further examinations.

Statistical analysis. Statistical analyses were performed using GraphPad Prism (version 7.0; GraphPad Software, Inc.). All quantitative data are presented as the mean \pm standard deviation. Differences between groups were determined using one-way ANOVA test with Tukey's post hoc test. $\mathrm{P}<0.05$ was considered to indicate a statistically significant difference. All experiments were repeated three times. 
Table II. Primers used for reverse transcription-quantitative PCR and their respective sequences.

\begin{tabular}{ll}
\hline Gene & \multicolumn{1}{c}{ Sequence (5'-3') } \\
\hline CCNB1 & F: CTTAGACAAATTCTGAACTAGTGTACA \\
& R: ATTCTTGACAACGGTGAAT \\
E-cadherin & F: GAGTCATCAGTGTGGTCACC \\
& R:GGGGGCATCAGCATCAGTCA \\
N-cadherin & F: AAGTGGGTTGAAGCGTATCACA \\
& R:ACACCGAGCTCAGCTACACTTG \\
p120-catenin & F: GCCAACTCAGCAGACATATCAC \\
& R:CCATCAAGAACCTACAGACTCC \\
GAPDH & F: GGCAGTGATGGCATGGACTGT \\
& R:CCTTCATTGACCTCAACTACA
\end{tabular}

CCNB1, cyclin B1; F, forward; R, reverse.

\section{Results}

CCNB1 expression is upregulated in pituitary adenomas and is higher in the invasive group. The present results revealed that the expression of CCNB1 was upregulated in tumor samples compared with the normal control (Fig. 1A). In addition, the experimental results of another group of tumor specimens proved that the expression of CCNB1 was markedly higher in the invasive group compared with the non-invasive group (Fig. 1B).

sh-CCNB1 downregulates the expression of CCNB1. Furthermore, it was demonstrated that CCNB1 expression was affected by the lentiviral-mediated shRNA infection. The infection effect was observed using fluorescent imaging of GFP-positive GH3 and MMQ cells following lentivirus transfection (Fig. 2A-F). The cells were transfected with the shRNA and after $72 \mathrm{~h}$, the transfection rates of shRNA-CCNB1 group and shRNA-NC group were both $\sim 80 \%$. The interference effect was validated via RT-qPCR and western blotting. These results revealed that CCNB1 was significantly decreased both at the mRNA and protein levels compared with the control and NC groups (Fig. 2G and H).

Knockdown of CCNB1 suppresses the proliferation of $\mathrm{GH} 3$ and $M M Q$ cells. In order to investigate the function of CCNB1 in the growth of pituitary adenoma, loss-of-function studies were performed with a CCK-8 assay for 3 days following lentiviral-mediated CCNB1 knockdown. GH3 and MMQ cells growth were inhibited in the shRNA-CCNB1 group in comparison with the shRNA-NC group (Fig. 2I). The present results indicated that downregulation of CCNB1 could inhibit the proliferation of pituitary adenoma cells.

Downregulation of CCNB1 induces cell cycle arrest and promotes apoptosis. Annexin V-FITC/PI flow cytometry was performed to investigate the rate of apoptosis. The results of the flow cytometry analysis revealed that the percentage of cells in the $G_{0} / G_{1}$ phase in the shRNA-CCNB1 group was significantly decreased, while a larger percentage of these cells were arrested at the $\mathrm{G}_{2} / \mathrm{M}$ phase compared with the $\mathrm{NC}$

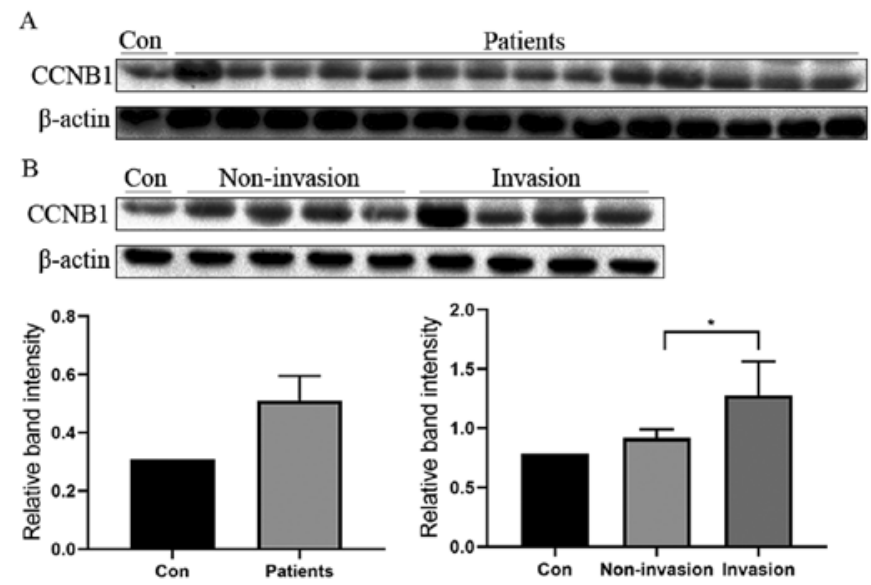

Figure 1. Protein expression levels of CCNB1 in pituitary tissues. The samples were divided into two experimental groups. (A) In the first group, the expression level of CCNB1 was analyzed in 14 pituitary adenoma tissue specimens and a normal pituitary gland. Expression levels of CCNB1 in normal $(n=1)$ and pituitary adenomas $(n=14)$ were detected by western blotting. (B) In the second group, four non-invasive, four invasive pituitary adenoma tissue specimens and a normal pituitary were investigated. Expression levels of CCNB1 in normal $(n=1)$ and non-invasive $(n=4)$ and invasive pituitary adenomas $(n=4)$ were detected by western blotting. Blots were applied to the same exposure condition. Band intensities were quantified and standardized to -actin for active CCNB1. ${ }^{*} \mathrm{P}<0.05$. CCNB1, cyclin B1; Con, pituitary tissue sample; patients, pituitary adenoma tissue samples.

group (Fig. 2J). In addition, compared with the NC group, the rate of apoptosis in the shRNA-CCNB1 group was significantly increased ( 0.08 vs. $54.81 \%$, respectively; Fig. $2 \mathrm{~K})$. The present results suggested that shRNA-CCNB1-mediated inhibition of cell proliferation was partially due to cell apoptosis and abnormal cell cycle progression.

Downregulation of CCNB1 inhibits the EMT. The present study further investigated whether changes in CCNB1 influenced the EMT using RT-qPCR. The results demonstrated that the mRNA expression of the epithelial cell markers E-cadherin and p120-catenin were significantly increased, and in contrast, the mesothelial cell marker N-cadherin was significantly decreased between the sh-CCNB1 group and the normal control (Fig. 2L). These results suggested that CCNB1 is involved in the process of EMT in pituitary adenoma cells.

RES inhibits the expression of CCNB1. The effect of RES on CCNB1 was analyzed using RT-qPCR and western blot analysis. The present results revealed that CCNB1 was significantly decreased following treatment with RES at different concentrations (100 and $300 \mu \mathrm{M})$, in line with the RT-qPCR results (Fig. 3A and $\mathrm{B}$, respectively).

RES suppresses cell proliferation and inhibits EMT. Downregulation of CCNB1 mediated by RES treatment significantly suppressed the growth rate of GH3 cells compared with the control group (Fig. 3C). The mRNA expression levels of the epithelial cell markers E-cadherin $(\mathrm{P}<0.001)$ and p120-catenin $(\mathrm{P}<0.001)$ were increased, and the mesothelial cell marker $\mathrm{N}$-cadherin $(\mathrm{P}<0.001)$ was decreased in the $100 \mu \mathrm{M}$ RES group. However, E-cadherin and p120-catenin expression is similar between the $300 \mu \mathrm{M}$ RES group and the control group (Fig. 3D). 


\section{A}

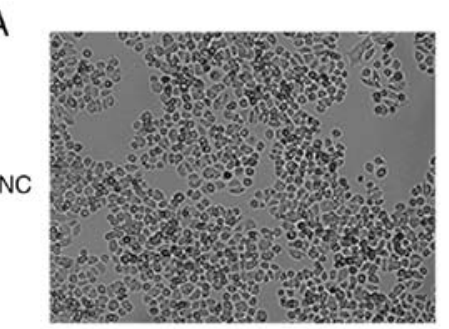

D

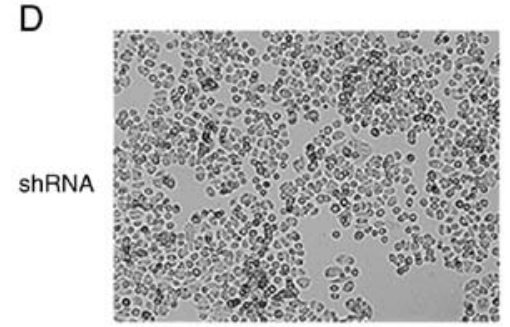

G

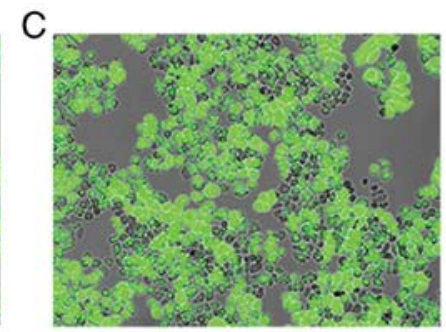

$\mathrm{F}$

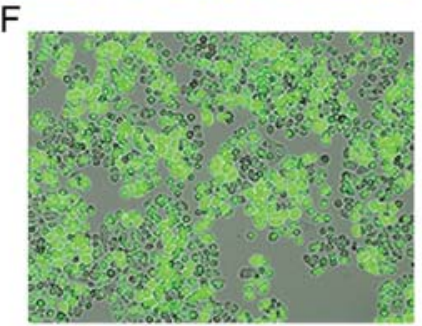

E

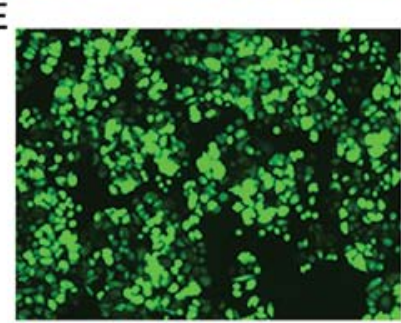

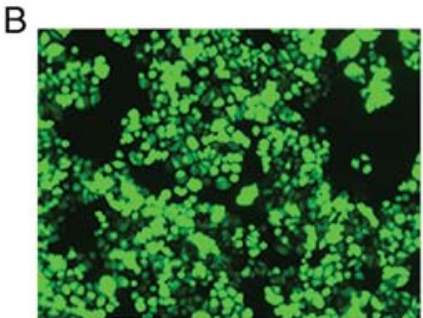

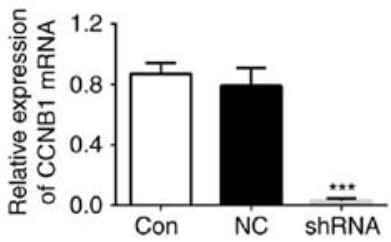

\section{믐on}

ShRNA
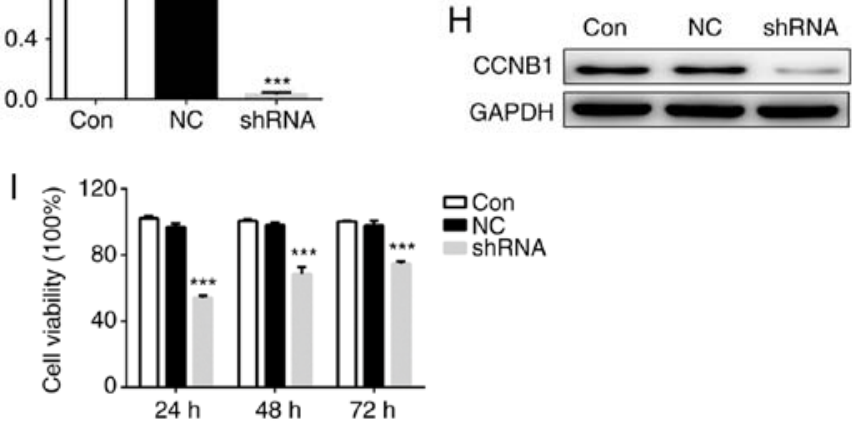

J
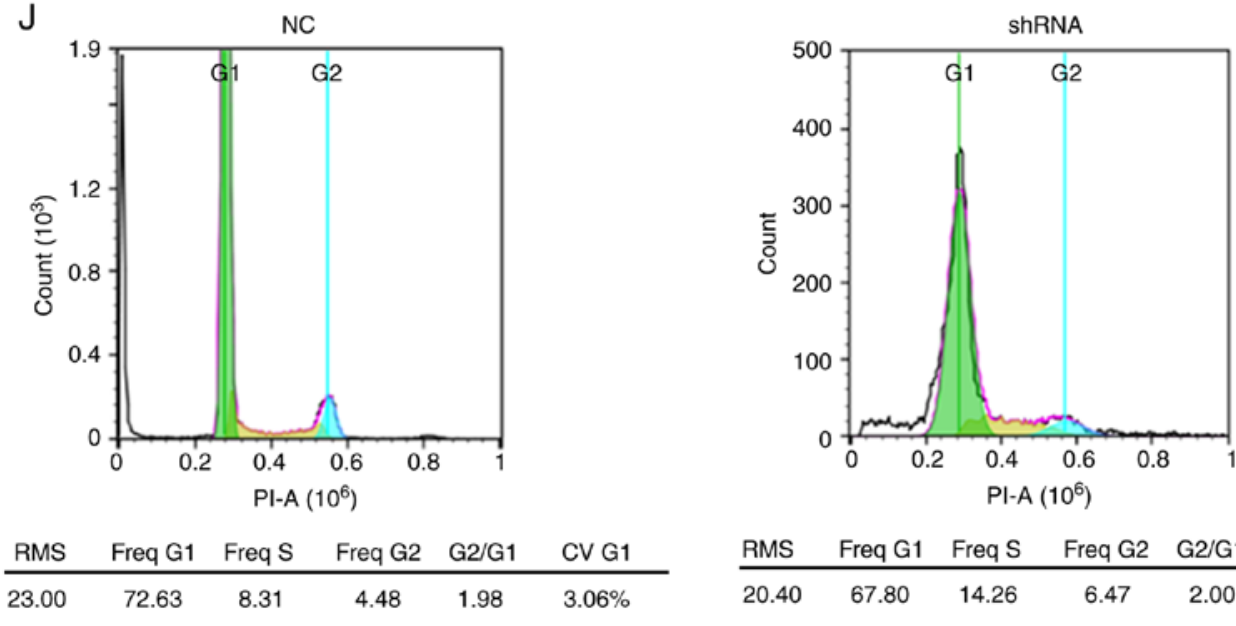

\begin{tabular}{cccccc} 
RMS & Freq G1 & Freq S & Freq G2 & G2/G1 & CV G1 \\
\hline 23.00 & 72.63 & 8.31 & 4.48 & 1.98 & $3.06 \%$
\end{tabular}

\begin{tabular}{cccccc} 
RMS & Freq G1 & Freq S & Freq G2 & G2/G1 & CV G1 \\
\hline 20.40 & 67.80 & 14.26 & 6.47 & 2.00 & $10.39 \%$
\end{tabular}
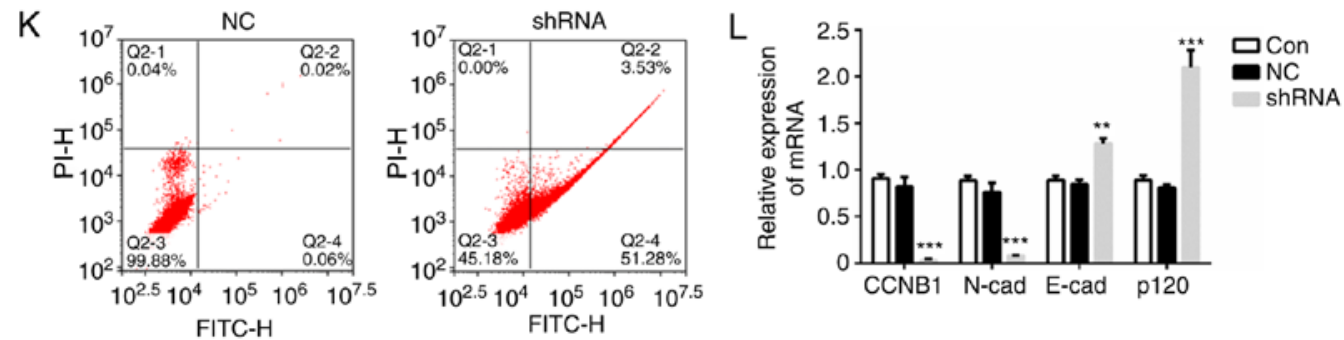

Figure 2. Effect of shRNA on CCNB1 expression, proliferation, cell cycle, apoptosis and EMT markers. (A) Brightfield, (B) fluorescent and (C) merged images of the NC group after $72 \mathrm{~h}$. (D) Brightfield, (E) fluorescent and (F) merged images of the CCNB1 shRNA group after $72 \mathrm{~h}$. (G) Quantification of mRNA expression levels of CCNB1 following lentiviral transfection by RT-qPCR. (H) Western blot analysis of the protein expression levels of CCNB1 in the CCNB1 shRNA group. (I) Proliferation of lentiviral transfected cells. (J) Percentage of cells in $\mathrm{G}_{1}$, $\mathrm{G}_{2} / \mathrm{M}$ or $\mathrm{S}$ phase was assessed after lentivirus transfection. (K) Annexin V-FITC/PI double staining flow cytometry was performed to evaluate apoptosis. (L) Quantification of mRNA expression levels via RT-qPCR in the shRNA group. ${ }^{* *} \mathrm{P}<0.01,{ }^{* * *} \mathrm{P}<0.001$ vs. NC. CCNB1, cyclin B1; RT-qPCR, reverse transcription-quantitative PCR; Con, blank control group; NC, negative control group; shRNA, short hairpin RNA lentivirus infection group; N-cad, N-cadherin; E-cad, E-cadherin; p120, p120-catenin; PI, propidium iodide. 
A

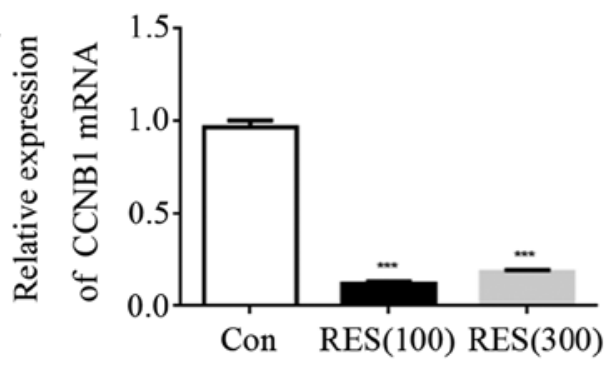

B

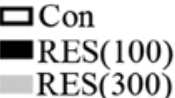

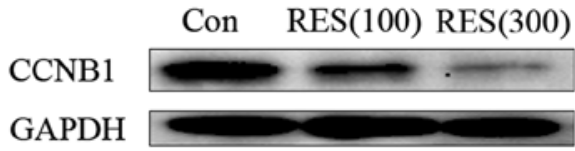

$\mathrm{C}$

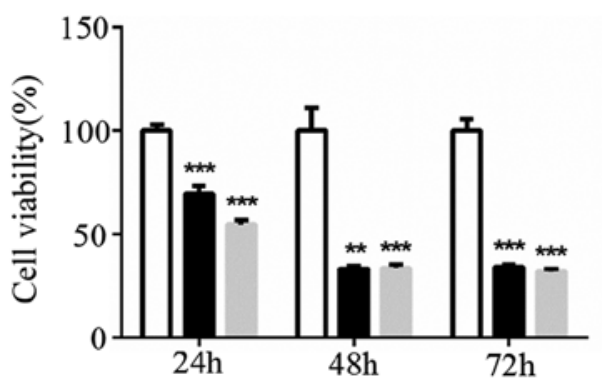

$\mathrm{D}$

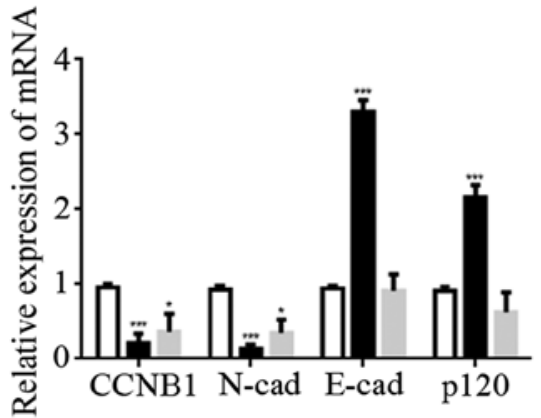

Figure 3. Effect of RES on CCNB1 expression, cell proliferation and EMT markers. (A) Quantification of mRNA expression levels of CCNB1 in the RES treatment group via RT-qPCR. (B) Western blotting images of the protein expression levels of CCNB1 in the RES treatment group. (C) Downregulation of CCNB1 by RES suppressed the cell proliferation following RES treatment. (D) Expression levels of mRNA of E-cad and p120 were increased, and the $\mathrm{N}$-cad was decreased when comparing the RES group and control group using RT-qPCR. ${ }^{*} \mathrm{P}<0.05 ;{ }^{* *} \mathrm{P}<0.01$ and ${ }^{* * *} \mathrm{P}<0.001$ vs. Con. RES, resveratrol; CCNB1, cyclin B1; RT-qPCR, reverse transcription-quantitative PCR; Con, blank control group; RES(100), $100 \mu \mathrm{M}$ RES group; RES(300), $300 \mu \mathrm{M}$ RES group; p120, p120-catenin; E-cad, E-cadherin; N-cad, N-cadherin.

\section{Discussion}

CCNB1, encoded by the CCNB1 gene, belongs to the cyclin superfamily (6). In a previous study, it was revealed that CCNB1 exhibited abnormal expression levels in tumor tissues through a meta analysis (16). Other previous studies have demonstrated that CCNB1 was closely associated with tumor progression and was highly expressed in tumor tissues and cells (31). Song et al (21) revealed that the high expression levels of CCNB1 promoted the proliferation of esophageal cells, enhanced cell migration, and led to a significant increase in the invasiveness of esophageal squamous cell carcinoma cells (21). In addition, abnormal expression levels of CCNB1 were demonstrated to be associated with tumor invasion, metastasis and prognosis in other studies $(32,33)$. Thus, CCNB1 was indicated as a pivotal target gene to promote the malignant phenotype and proliferation of tumors $(34,35)$. However, the molecular mechanism underlying CCNB1-mediated promotion for tumor cell invasion remains unknown. Therefore, the present study aimed to examine the role of CCNB1 in the development of pituitary adenomas. Using clinical samples, it was revealed that the expression levels of CCNB1 increased in tumor samples exhibiting increasing tumor invasion potential, and the levels of CCNB1 were significantly higher compared with the non-invasive group, which was consistent with a previous study (17). The present results indicated that the high expression levels of CCNB1 were associated with the invasion degree of pituitary adenoma. In addition, knockdown of CCNB1 using a lentivirus-packaged shRNA designed in the present study could arrest the proliferation of pituitary adenoma cells at the $\mathrm{G}_{2} / \mathrm{M}$ phase, and also induce cell apoptosis. The present results suggested that CCNB1 was associated with pituitary adenoma cell proliferation and mediated cell cycle.
EMT is a biological process characterized by a loss of polarization and cell-cell adhesion, and a gain of fibroblastoid phenotype and increased cell motility $(36,37)$. Recent studies have demonstrated that EMT plays an important role in tumor invasiveness. Previous research has reported that cyclin A2 deficiency promoted cell invasion in fibroblasts, and they also revealed that silencing of cyclin A2 in normal breast cancer epithelial cells significantly promoted EMT $(38,39)$. Wang et al (19) reported that ADAM12 can induce the invasion, migration and EMT of pituitary tumor cells through the EGFR/ERK pathway. The EMT process was regulated by key EMT mediators and resulted in a switch from E-cadherin to N-cadherin (40). p120-catenin is a multifunctional protein that is bound to E-cadherin on the cell membrane, and its dissociation leads to E-cadherin degradation (41). Jia et al (42) examined the expression levels of EMT biomarkers in 95 human pituitary tumors and revealed that E-cadherin and $\mathrm{N}$-cadherin were valuable biomarkers in assessing the clinical course of pituitary adenomas. In the present study, it was revealed that knockdown of CCNB1 significantly decreased the expression levels of the mesothelial cell marker $\mathrm{N}$-cadherin, whereas the expression of epithelial cell markers E-cadherin and p120-catenin were significantly increased following lentivirus transfection. The present results demonstrated that CCNB1 downregulation inhibited EMT in pituitary adenomas. Therefore, the present results suggested that CCNB1 may activate EMT.

A previous study has demonstrated that RES inhibits cell proliferation and decreased prolactin level via estrogen receptors (43). A number of studies have demonstrated that RES oligomers can be used for the prevention and treatment of different types of cancer, for example, lung cancer, colon cancer and hepatoma (44-46). In the present study, the expression levels 
of CCNB1 were significantly decreased with the addition of RES at different concentrations (100 and $300 \mu \mathrm{M})$. Furthermore, downregulation of CCNB1 mediated by RES significantly suppressed the growth and proliferation of GH3 and MMQ cells. These results demonstrated that RES could downregulate the expression levels of CCNB1, affecting the cell cycle. These results were consistent with the lentiviral transfection that inhibited cell proliferation. Therefore, RES could be used as an inhibitor to suppress the proliferation of pituitary tumor cells. The mRNA expression levels of the epithelial cell markers E-cadherin and p120-catenin were increased whereas the mesothelial cell marker $\mathrm{N}$-cadherin was decreased in the $100 \mu \mathrm{M}$ RES group. In addition, E-cadherin and p120-catenin expression were similar between the $300 \mu \mathrm{M}$ RES group and the control group. The results indicate that $100 \mu \mathrm{M}$ RES can affect the EMT process by reducing the expression of CCNB1. However, we hypothesize that excessive RES may directly affect the EMT process, in addition to inhibiting the expression of CCNB1. In future research, the specific mechanisms by which resveratrol affects the EMT process of pituitary tumors will be explored.

In summary, the present study suggested that suppressing the CCNB1 gene may regulate the proliferation and apoptosis of pituitary tumor cells and activate EMT. The present results may improve the current understanding of the biological mechanisms of CCNB1 in the development and progression of pituitary adenoma. However, further studies are required in order to confirm these results and to investigate the potential pathway involved in the development of pituitary adenomas. It was also observed that RES inhibited the expression levels of CCNB1 in pituitary tumor cells, affecting cell proliferation and EMT. The present results suggested that RES may suppress the expression level of CCNB1 and may represent a novel clinical treatment for pituitary adenomas.

\section{Acknowledgements}

The authors would like to thank Ms Hong-Yun Wang (Department of Cell and Biology, Beijing Neurosurgical Institute) for the collection of the pituitary adenoma samples and Professor Gui-Lin Li for the pathology results (Department of Pathology, Beijing Tiantan Hospital).

\section{Funding}

The present study was funded by the Beijing Natural Science Foundation (grant no. 7162034).

\section{Availability of data and materials}

All data generated or analyzed during the present study are included within this published article.

\section{Authors' contributions}

PZ designed and supervised the project. BL and HBZ performed the majority of the experiments and drafted the manuscript. GDS and JHC performed the experiments, acquired the data and wrote the article. CZL and YZZ designed the experiments and revised the manuscript. All authors read and approved the final manuscript.

\section{Ethics approval and consent to participate}

The present study was approved by the Ethics Committee of Beijing Tiantan Hospital, Capital Medical University (Beijing, China).

\section{Patient consent for publication}

Not applicable.

\section{Competing interests}

The authors declare that they have no competing interests.

\section{References}

1. McNeill KA: Epidemiology of brain tumors. Neurol Clin 34: 981-998, 2016.

2. Scoazec JY, Couvelard A and Reseau T: Classification of pancreatic neuroendocrine tumours: Changes made in the 2017 WHO classification of tumours of endocrine organs and perspectives for the future. Ann Pathol 37: 444-456, 2017 (In French).

3. Raverot G, Burman P, McCormack A, Heaney A, Petersenn S, Popovic V, Trouillas J and Dekkers OM; European Society of Endocrinology: European society of endocrinology clinical practice guidelines for the management of aggressive pituitary tumours and carcinomas. Eur J Endocrinol 178: G1-G24, 2018.

4. Lenders $\mathrm{N}$ and McCormack A: Malignant transformation in non-functioning pituitary adenomas (pituitary carcinoma). Pituitary 21: 217-229, 2018.

5. Lopes MBS: The 2017 World Health Organization classification of tumors of the pituitary gland: A summary. Acta Neuropathol 134: 521-535, 2017.

6. Miyazaki T and Arai S: Two distinct controls of mitotic cdk1/cyclin B1 activity requisite for cell growth prior to cell division. Cell Cycle 6: 1419-1425, 2007.

7. Takizawa CG and Morgan DO: Control of mitosis by changes in the subcellular location of cyclin-B1-Cdk1 and Cdc25C. Curr Opin Cell Biol 12: 658-665, 2000.

8. Strauss B, Harrison A, Coelho PA, Yata K, Zernicka-Goetz M and Pines J: Cyclin B1 is essential for mitosis in mouse embryos, and its nuclear export sets the time for mitosis. J Cell Biol 217: 179-193, 2018.

9. Mussnich P,Raverot G, Jaffrain-Rea ML, Fraggetta F, Wierinckx A, Trouillas J, Fusco A and D'Angelo D: Downregulation of miR-410 targeting the cyclin B1 gene plays a role in pituitary gonadotroph tumors. Cell Cycle 14: 2590-2597, 2015.

10. Pandey JP, Kistner-Griffin E, Namboodiri AM, Iwasaki M, Kasuga Y, Hamada GS and Tsugane S: Higher levels of antibodies to the tumour-associated antigen cyclin B1 in cancer-free individuals than in patients with breast cancer. Clin Exp Immunol 178: 75-78, 2014.

11. Nimeus-Malmström E, Koliadi A, Ahlin C, Holmqvist M, Holmberg L, Amini RM, Jirström K, Wärnberg F, Blomqvist C, Fernö M and Fjällskog ML: Cyclin B1 is a prognostic proliferation marker with a high reproducibility in a population-based lymph node negative breast cancer cohort. Int J Cancer 127: 961-967, 2010.

12. Kreis NN, Sanhaji M, Krämer A, Sommer K, Rödel F, Strebhardt K and Yuan J: Restoration of the tumor suppressor p53 by downregulating cyclin B1 in human papillomavirus 16/18-infected cancer cells. Oncogene 29: 5591-5603, 2010.

13. Yoshida T, Tanaka S, Mogi A, Shitara Y and Kuwano H: The clinical significance of Cyclin B1 and Wee1 expression in non-small-cell lung cancer. Ann Oncol 15: 252-256, 2004.

14. Nozoe T, Korenaga D, Kabashima A, Ohga T, Saeki H and Sugimachi K: Significance of cyclin B1 expression as an independent prognostic indicator of patients with squamous cell carcinoma of the esophagus. Clin Cancer Res 8: 817-822, 2002.

15. Kedinger V, Meulle A, Zounib O, Bonnet ME, Gossart JB, Benoit E, Messmer M, Shankaranarayanan P, Behr JP, Erbacher P, et al: Sticky siRNAs targeting survivin and cyclin B1 exert an antitumoral effect on melanoma subcutaneous xenografts and lung metastases. BMC Cancer 13: 338, 2013. 
16. Zhao P, Hu W, Wang H, Yu S, Li C, Bai J, Gui S and Zhang Y: Identification of differentially expressed genes in pituitary adenomas by integrating analysis of microarray data. Int $\mathbf{J}$ Endocrinol 2015: 164087, 2015.

17. Zhao P, Zhang PF, Hu W, Wang H, Yu G, Wang Z, Li C, Bai J and Zhang Y: Upregulation of cyclin B1 plays potential roles in the invasiveness of pituitary adenomas. J Clin Neurosci 43: 267-273, 2017.

18. Vu T and Datta PK: Regulation of EMT in colorectal cancer: A culprit in metastasis. Cancers (Basel) 9: E171, 2017.

19. Wang JW, Zhang Z, Li R, Mao F, Sun W, Chen J, Zhang H, Bartsch JW, Shu K and Lei T: ADAM12 induces EMT and promotes cell migration, invasion and proliferation in pituitary adenomas via EGFR/ERK signaling pathway. Biomed Pharmacother 97: 1066-1077, 2018.

20. Forte E, Chimenti I, Rosa P, Angelini F, Pagano F, Calogero A Giacomello A and Messina E: EMT/MET at the crossroad of stemness, regeneration and oncogenesis: The Ying-Yang equilibrium recapitulated in cell spheroids. Cancers 9: E98, 2017.

21. Song Y, Zhao C, Dong L, Fu M, Xue L, Huang Z, Tong T, Zhou Z, Chen A, Yang Z, et al: Overexpression of cyclin B1 in human esophageal squamous cell carcinoma cells induces tumor cell invasive growth and metastasis. Carcinogenesis 29: 307-315, 2008.

22. Singh SK, Banerjee S, Acosta EP, Lillard JW and Singh R: Resveratrol induces cell cycle arrest and apoptosis with docetaxel in prostate cancer cells via a p53/p21 (WAF1/CIP1) and p27(KIP1) pathway. Oncotarget 8: 17216-17228, 2017.

23. Medina-Aguilar R, Marchat LA, Arechaga Ocampo E, Gariglio P, García Mena J, Villegas Sepúlveda N, Martínez Castillo M and López-Camarillo C: Resveratrol inhibits cell cycle progression by targeting Aurora kinase A and Polo-like kinase 1 in breast cancer cells. Oncol Rep 35: 3696-3704, 2016.

24. de Freitas Silva M, Coelho LF, Guirelli IM, Pereira RM, Ferreira-Silva GÁ, Graravelli GY, Horvath RO, Caixeta ES Ionta $\mathrm{M}$ and Viegas C: Synthetic resveratrol-curcumin hybrid derivative inhibits mitosis progression in estrogen positive MCF-7 breast cancer cells. Toxicol In Vitro 50: 75-85, 2018.

25. Wang G, Guo X, Chen H, Lin T, Xu Y, Chen Q, Liu J, Zeng J, Zhang XK and Yao X: A resveratrol analog, phoyunbene B, induces $\mathrm{G} 2 / \mathrm{M}$ cell cycle arrest and apoptosis in HepG2 liver cancer cells. Bioorg Med Chem Lett 22: 2114-2118, 2012.

26. Hardy $\mathrm{J}$ and Vezina JL: Transsphenoidal neurosurgery of intracranial neoplasm. Adv Neurol 15: 261-273, 1976.

27. Wilson CB: A decade of pituitary microsurgery. The Herbert Olivecrona lecture. J Neurosurg 61: 814-833, 1984.

28. Knosp E, Steiner E, Kitz K and Matula C: Pituitary adenomas with invasion of the cavernous sinus space: A magnetic resonance imaging classification compared with surgical findings. Neurosurgery 33: 610-618, 1993.

29. Livak KJ and Schmittgen TD: Analysis of relative gene expression data using real-time quantitative PCR and the 2(-Delta Delta C(T)) method. Methods 25: 402-408, 2001

30. Joe AK, Liu H, Suzui M, Vural ME, Xiao D and Weinstein IB: Resveratrol induces growth inhibition, S-phase arrest, apoptosis, and changes in biomarker expression in several human cancer cell lines. Clin Cancer Res 8: 893-903, 2002

31. Zhou SL, Yue WB, Fan ZM, Du F, Liu BC, Li B, Han XN, $\mathrm{Ku}$ JW, Zhao XK, Zhang P, et al: Autoantibody detection to tumor-associated antigens of P53, IMP1, P16, cyclin B1, P62, C-myc, Survivn and Koc for the screening of high-risk subjects and early detection of esophageal squamous cell carcinoma. Dis Esophagus 27: 790-797, 2014.
32. Fang Y, Liang X, Jiang W, Li J, Xu J and Cai X: Cyclin B suppresses colorectal cancer invasion and metastasis by regulating E-cadherin. PLoS One 10: e0126875, 2015.

33. Lee JH, Lee HJ, Sim DY, Jung JH, Kim KR and Kim SH: Apoptotic effect of lambertianic acid through AMPK/FOXM1 signaling in MDA-MB231 breast cancer cells. Phytother Res 32: 1755-1763, 2018.

34. Li L, Yang Y, Wu M, Yu Z, Wang C, Dou G, He H, Wang H, Yang N, Qi $\mathrm{H}$ and $\mathrm{Xu} \mathrm{X}$ : $\beta$-asarone induces apoptosis and cell cycle arrest of human glioma U251 cells via suppression of HnRNP A2/B1-mediated pathway in vitro and in vivo. Molecules 23: E1072, 2018.

35. Lu M, Breyssens H, Salter V, Zhong S, Hu Y, Baer C, Ratnayaka I, Sullivan A, Brown NR, Endicott J, et al: Restoring p53 function in human melanoma cells by inhibiting MDM2 and Cyclin B1/CDK1-phosphorylated nuclear iASPP. Cancer Cell 30: 822-823, 2016

36. Lamouille S, Xu J and Derynck R: Molecular mechanisms of epithelial-mesenchymal transition. Nat Rev Mol Cell Bio 15: 178-196, 2014.

37. Gonzalez DM and Medici D: Signaling mechanisms of the epithelial-mesenchymal transition. Sci Signal 7: re8, 2014.

38. Bendris N, Cheung CT, Leong HS, Lewis JD, Chambers AF, Blanchard JM and Lemmers B: Cyclin A2, a novel regulator of EMT. Cell Mol Life Sci 71: 4881-4894, 2014.

39. Arsic N, Bendris N, Peter M, Begon-Pescia C, Rebouissou C, Gadéa G, Bouquier N, Bibeau F, Lemmers B and Blanchard JM: A novel function for Cyclin A2: Control of cell invasion via RhoA signaling. J Cell Biol 196: 147-162, 2012.

40. Rastogi I, Rajanna S, Webb A, Chhabra G, Foster B, Webb B and Puri N: Mechanism of c-Met and EGFR tyrosine kinase inhibitor resistance through epithelial mesenchymal transition in non-small cell lung cancer. Biochem Bioph Res Commun 477: 937-944, 2016.

41. Kourtidis A, Ngok SP and Anastasiadis PZ: p120 Catenin: An essential regulator of cadherin stability, adhesion-induced signaling, and cancer progression. Prog Mol Biol Transl Sci 116: 409-432, 2013

42. Jia W, Zhu JK, Martin TA, Jiang AH, Sanders AJ and Jiang WG: Epithelial-mesenchymal Transition (EMT) markers in human pituitary adenomas indicate a clinical course. Anticancer Res 35 2635-2643, 2015.

43. Wang $\mathrm{C}, \mathrm{Hu} \mathrm{ZQ}$, Chu $\mathrm{M}$, Wang Z, Zhang WG, Wang LZ Li CG and Wang JS: Resveratrol inhibited GH3 cell growth and decreased prolactin level via estrogen receptors. Clin Neurol Neurosur 114: 241-248, 2012.

44. Xue YQ, Di JM, Luo Y, Cheng KJ, Wei X and Shi Z: Resveratrol oligomers for the prevention and treatment of cancers. Oxid Med Cell Longev 2014: 765832, 2014.

45. Yousef M, Vlachogiannis IA and Tsiani E: Effects of resveratrol against lung cancer: In vitro and in vivo studies. Nutrients 9: pii: E1231, 2017.

46. Nana AW, Chin YT, Lin CY, Ho Y, Bennett JA, Shih YJ, Chen YR, Changou CA, Pedersen JZ, Incerpi S, et al: Tetrac downregulatescatenin and HMGA2 to promote the effect of resveratrol in colon cancer. Endocr Relat Cancer 25: 279-293, 2018.

(i) (-) This work is licensed under a Creative Commons Attribution-NonCommercial-NoDerivatives 4.0 International (CC BY-NC-ND 4.0) License. 